\title{
Research on the desktop sharing mechanism of online teaching system
}

\author{
Xiaoyun Liu, a , Jian Shen ${ }^{2, b}$ \\ ${ }^{1}$ School of foreign languages, Jiangxi Science \& Technology Normal University, Fenglin Avenue \\ 605\#, Nanchang, Jiangxi P.R.China 330013 \\ ${ }^{2}$ School of Civil Engineering and Architecture, Jiangxi Science \& Technology Normal University, \\ Fenglin Avenue 605\#, Nanchang, Jiangxi P.R.China 330013 \\ a505132338@qq.com, b894282069@qq.com
}

Key words: desktop sharing mechanism, online teaching system; screenshot function

Abstract: It is essential to obtain much information, such as text, audio and video, in online teaching. Based on the presenter's desktop screenshot function, an efficient mechanism of desktop sharing mechanism is designed. The mechanism can be used to help teachers to share their desktop and the operation of the desktop. With this mechanism, the explanation and demonstration form of courseware is more rich to adapt to different teaching contents and characteristics of curriculum.

\section{Introduction}

The desktop sharing function can be used to provide a more full of variety way for demonstration teaching[1,2]. In addition to the presentation, the presenter can also let others see his computer desktop and all the operations on the desktop directly including all kinds of documents or software demonstration and so on[3].

\section{Desktop Sharing Mechanism}

The desktop sharing function is different from the document one, so it is needed to develop some module separately to support this function. To relay the desktop sharing action, a method which is similar to the way of Flash animation is selected[4]. The basic principle of the mechanism is calling the presenter's desktop screenshot function, transcoding continuously capture key frames and key frames in the smaller interval to form video and transferring to the viewer client. To reduce the amount of data, only the key frames and real-time mouse position is transferred and the other frames are simulated according to the key frames.

There are three main parts in the desktop sharing mechanism, Applet, Server and Flash. The Applet is referred to programs written in Java language, which can be included in the HTML page and be loaded in each user client to part and code a local desktop picture. While the Server end file blocks of code is used to decode and restructure video streaming form and transmit to each Flash client. Through the above methods, the effect of synchronous sharing is achieved. The realization of desktop sharing mechanism is shown in Fig.1. 


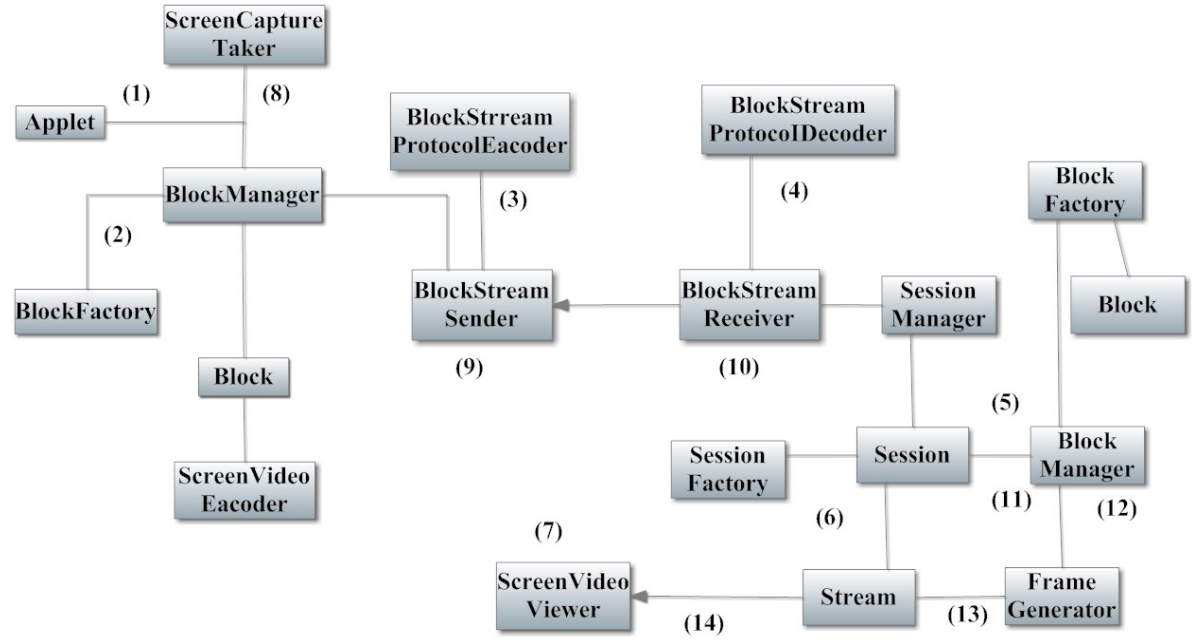

Fig. 1 desktop sharing mechanism

\section{Basic Flow}

The desktop sharing mechanism design is shown as figure 2 and the detailed process is shown as follow ones:

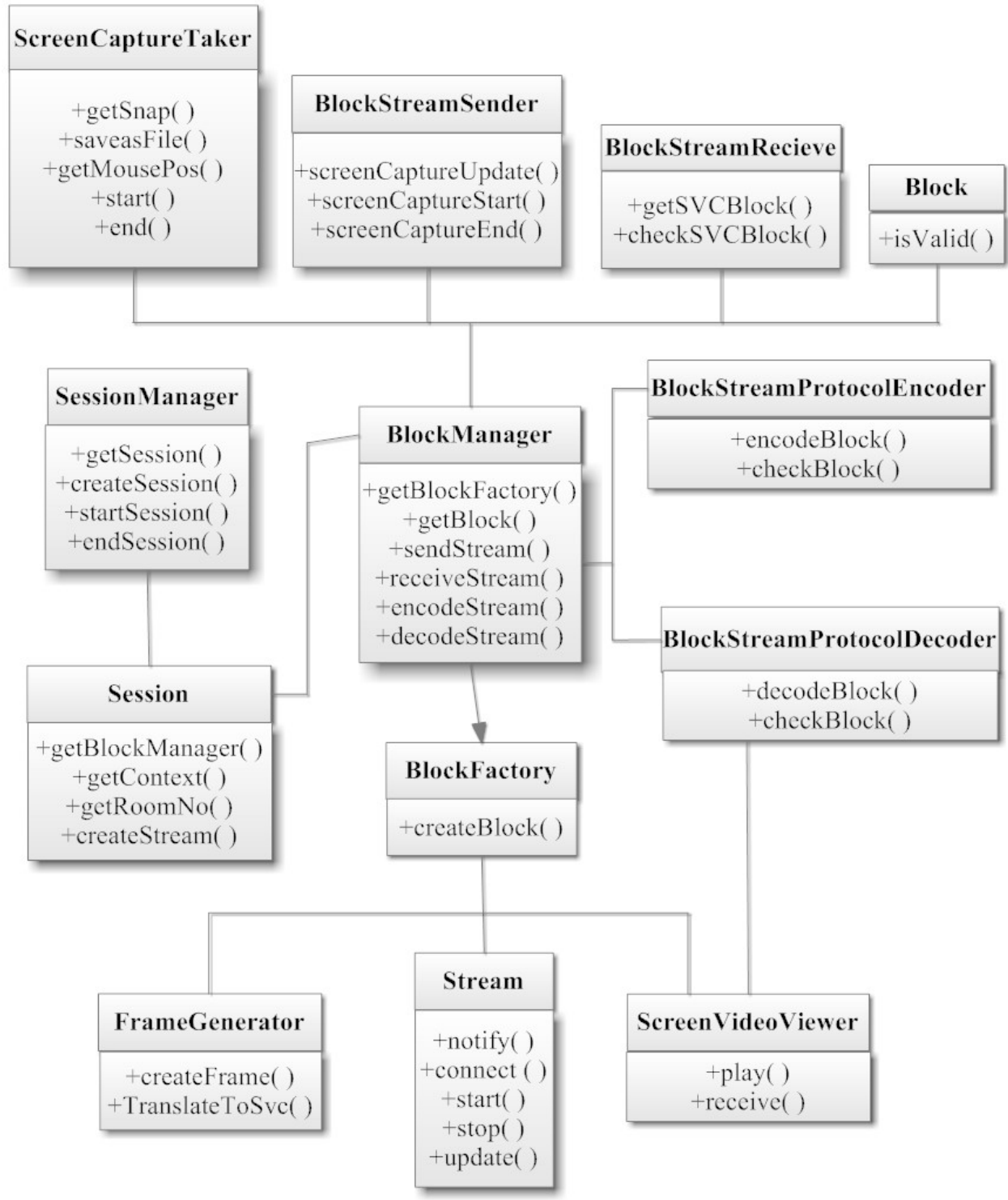

Fig.2 Desktop sharing mechanism of class diagram 
1) BlockManager module is called by Applet to transfer the screen size.

2) BlockFactory is invoked by BlockManager to create Screen Video Codec (SVC) Block format.

3) ScreenCaptureStart messages are sent to the server by BlockStreamSender.

4) the received messages are decoded (removed the RTMP protocol format) and forwarded to SessionManager by BlockStreamReceiver.

5) A Session is created by SessionManager, then a piece of manager BlockManager is created by the Session to call a BlockFactory. A.Block is created by BlockFactory to save the SVC format of the data from the client.

6) A Stream is created by the Session to inform the client to share and transmit screen sharing flow.

7) In other clients, desktop sharing flow ise broadcast by ScreenVideoViewer.

8) In the process of sharing, a snapshot of the screen is grabbed by ScreenCaptureTaker continuly and the latest Blocks are updated by BlockManager constantly.

9) if the Block in BloackManager is changing, ScreenVideoEncoder will be called by BlockStreamSender to code the latest screen shot and the Block will be encapsulated as a packet by ScreenCaptureUpdate. In the end, the packet is sent to the server.

10) Receiving the packet, the date is decoded and sent to SessionManager by BlockStreamReceiver.

11) The date is put forward BlockManager and stored to the corresponding Block by Session.

12) FrameGenerator is called by BlockManager periodically to generate SVC ( Screen Video

Codec ) from Block data frames.

13) The SVC frames is sent to the Stream and shared to the client by FrameGenerator.

14) SVC frame is played by ScreenVideoViewer.

\section{Session classes}

Session classes is the core of desktop sharing. It is established by SessionFactory and contains BlockManager, FrameGenerator, Stream, ScreenVideoViewer class. A new Session will be created by each user initiated desktop sharing and a Shared streaming video is established.

The Session class contains FrameGenerator class that the function of this class is encoding data blocks into one key frames and translating SVC data to transmit and play. The Session calss also contains Stream which is established between the client and server video class and connected, opened and closed video Stream by the connect(), start () and stop () method. The update method is used to update the video data.

The session class insides a very important BlockManager class. The BlockManager is used to manage the generation of data blocks, encoding, decoding, and the corresponding sending and receiving, etc. It needs to use screen scraping class ScreenCaptureTaker to grab a screenshot through getSnap () method and save the snapshot data by saveAsFile () method. At the same time, it also can obtain the current mouse position information of the screen.

BlockManager consists of BlcokFactory, BlockStreamSender, BlockStreamReceiver, BlockStream ProtocolEncoder and BlockStream ProtocolDecoder. The snapshot and mouse position data is encapsulated as a Block by BlcokFactory. The block data is sent and updated constantly by BlockStreamSender. The block data is obtained by BlockStreamReceiver through checkSvcBlock () 
method. The data transmission data block is coded and decoded by BlockStream ProtocolEncoder and BlockStream ProtocolDecoder.

\section{Conclusion}

The desktop sharing mechanism is provided by taking a screen capture video key frames and analog convert video streaming technology solutions.

Desktop sharing mechanism is given the specific process and the class diagram.

\section{Acknowledgments}

This research was supported by 2014 field scientific research subject "learning attitude research ESL students under the environment of culture" Funds of Jiangxi Science \& Technology Normal University (300098010626), which is gratefully acknowledged.

\section{References}

[1] Kim Needy. Distance Education: On the Use of Video Conferencing at the University of Pittsburgh [J]. Frontiers in Education Conference, 2007(11) : 12-18.

[2] Zhenguo Gao, Shaobin Cai. An Implementation of an Interactive Virtual Classroom based on Flash Media Server [J]. Computer applications in Engineering Education, 2012, 20(4):10-14. (in Chinese)

[3] Ali Begen, Tankut Akgul. Watching Video over the Web Partl: Streaming Protoeal [J]. Internet Computing, 2011, 02:53-62.

[4] Sila Chunwijitra, Arjulie John Berena. Authoring Tool based on Flash Technology for Web ELS Learning System to Support Higher Education[J]. Knowledge-Based Software Engineering,2011,111(282) : 112-115.

[5] Rong Liu, Zuxun Zhang, Jianqing Zhang. Multi-image Matching Technology and its Application of Investigation of Traffic Accident[J]. Information Technology and Applications, 2009(1) : 5-10. 